\title{
Factors influencing positive subjective health awareness in multicultural adolescents in South Korea: Data from the multicultural adolescents panel study
}

\author{
Sun Yeob Choi \\ Registered Nurse, Ewha Womans University Medical Center, Seoul · Graduate Student, College of Nursing, Ewha Womans University, Seoul, Korea
}

Purpose: This study aimed to identify the factors influencing positive subjective health awareness of middle school-age multicultural adolescents in Korea. Methods: This study used data from the fourth to sixth waves of the Multicultural Adolescents Panel Study conducted by the National Youth Policy Institute. In this study, a panel logit model analysis was performed using the Eviews 8.0 program $(p<.050)$. Results: Socioeconomic status $(\mathrm{r}=.02, \mathrm{t}=2.13, p=.033)$, body satisfaction $(\mathrm{r}=.71, \mathrm{t}=1.97, p=.048)$, and depression $(\mathrm{r}=-.74, \mathrm{t}=2.35, p=.018)$ influenced positive subjective health awareness in multicultural adolescents. When multicultural characteristics were entered into a statistical model with environmental and personal characteristics, multicultural characteristics did not have a significant influence on positive subjective health awareness. Conclusion: Environmental, personal, and multicultural characteristics were related to positive subjective health awareness in multicultural adolescents; thus, an integrated approach considering those variables is recommended. Nursing interventions and educational programs are needed to improve the psychological status of multicultural adolescents and reduce prejudice about them. In addition, nurses need to be sensitive to multicultural characteristics and the developmental characteristics of multicultural adolescents.

Key words: Health; Awareness; Adolescent; Cultural diversity

\section{Corresponding author \\ Sun Yeob Choi}

Ewha Womans University Medical Center, 1071 Anyangcheon-ro, Yangcheon-gu,

Seoul 07985, Korea

TEL: +82-2-2650-5114

FAX: +82-2-2655-0984

E-MAIL: ck9309@naver.com

Received Jun 26, 2021

Revised Aug 15, 2021

Accepted Sep 13, 2021

\section{INTRODUCTION}

With the steady increase in marriage immigrants in South Korea (hereafter, Korea), the number of multicultural children and adolescents born to them is increasing rapidly [1,2]. The total number of children and adolescents in Korea is decreasing, but the number of children and adolescents from multicultural families increased to 122,000 in 2019 and is expected to grow in the future [3]. Currently, about $80 \%$ of children in multicultural families are in childhood, but they will enter adolescence soon [1,2]. Multicultural adolescents experience dual-cultural stress and discrimination due to their multicultural background, along with other psychological vulnerabilities of adolescence such as identity confusion and role conflict [1,2].

In particular, multicultural adolescents of middle school age experience a higher level of maladaptation than those in other life stages [1]. At this age, multicultural adolescents experience the stress of multiculturalism [1,2] as they perform tasks, belong to peer groups, and build a sense of identity $[1,4]$. They also may experience health problems such as a lack of sleep and exercise and an inappropriate diet associated with the transition to middle school [5]. Multicultural adolescents have more experiences of smoking, drinking [6,7], depression, and suicidal thoughts [6,7] and perceive their subjective health status and well-being level negatively compared to non-multicultural adolescents [6,8].

Subjective health awareness is a self-evaluated health concept that represents an individual's perceived health level, and it is widely used to predict clinical health conditions and mortality [9-11]. Subjective health awareness encompasses health conditions and behavior from the past to present and predicts future health conditions $[4,10]$. Adolescence is a time when people are exposed to health risk factors and have the 
right to make decisions about their health, so they collect health-related information and make efforts to promote their health [4]. Therefore, subjective health awareness in adolescence affects various aspects of their lifestyle and behavior such as physical activity, eating habits, smoking, and drinking, which influence health conditions in adulthood $[4,9]$. Thus, it is important to identify the subjective health awareness of adolescents whose health habits have not been fixed. In prior studies, subjective health awareness of adolescents was found to be related to gender [4,11,12], family structure [9], residential area [11], economic state [9,11], psychological factors $[9,11,12]$, health behavior $[4,9,12]$ and relationships with peers $[4,12]$. However, a limitation of prior studies is that they were cross-sectional studies that identified correlations among variables [9] and case studies conducted on a small number of subjects [13]. In addition, most studies about subjective health awareness were conducted mainly on schoolage children [13] and there is a lack of research on subjective health awareness in multicultural adolescents with a comprehensive consideration of relevant factors [9].

Ethnicity and cultural background affect an individual's health perception and behaviors [14]. In addition, the mechanisms of recognizing and accepting health problems are different according to ethnic and racial identities [14]. Due to Korea's mono-ethnic and mono-cultural environment, many multicultural adolescents are exposed to prejudice and discrimination, causing them to experience mental and physical stress $[7,15]$. Subjective health awareness is a judgment about one's health, including behavioral, psychological, and social factors [10], and the well-being and health of multicultural adolescents are linked to individual, environmental, and cultural factors [8]. Thus, personal, environmental, and cultural characteristics should be considered in order to investigate the subjective health awareness of multicultural adolescents, who have distinctive experiences due to their multicultural background. Furthermore, it is necessary to identify factors affecting subjective health awareness in multicultural adolescents in order to develop interventions aiming to improve subjective health awareness. Thus, this study used the data of the Multicultural Adolescents Panel Study (MAPS) [16] to identify factors affecting positive subjective health awareness in multicultural adolescents. This study attempted to provide basic data for the development of nursing interventions and educational programs to improve the subjective health awareness of multicultural adolescents.

\section{METHODS}

Ethics statement: This study was approved by the Institutional Review Board of Ewha Womans University (No. Ewha-202105-0014-01).

\section{Study Design}

This study used a panel logit model to identify the factors affecting positive subjective health awareness of multicultural adolescents of middle school age by analyzing raw data from the MAPS [16].

\section{Data Source and Study Population}

The MAPS is a survey organized by the National Youth Policy Institute to track the development of multicultural adolescents. Since 2011, annual surveys have been conducted among a sample of multicultural children who were in fourth grade of elementary school at the start of the survey and their mothers [16]. MAPS is representative since it surveys multicultural adolescents from 2,537 schools in 16 cities and provinces of Korea [16]. The MAPS is meaningful since it investigates the environmental, personal, and multicultural factors that affect the growth and development of multicultural adolescents from a time-series perspective [16]. This study was conducted after receiving permission for data use from the MAPS and approval from the institutional review board of the researcher's affiliated institution (Ewha-202105-0014-01). This study used raw data from the fourth wave (2014) to the sixth wave (2016), when the subjects were at middle school age, and the homogeneity of the collected variables was maintained. Multicultural adolescents are defined as children from international marriage families, immigrant youth, and children from foreign countries [16]. However, most subjects of the MAPS were children from international marriage families [16]. The number of subjects was 1,380 in the fourth wave (2014), 1,347 in the fifth wave (2015), and 1,329 in the sixth wave (2016). In this study, a panel logit model analysis was performed on 1,329 subjects who participated in the survey up to the sixth wave.

\section{Study Variables}

\section{1) Dependent variable}

The dependent variable of this study was subjective health awareness, which was measured on a 4 point Likert scale (very healthy: 1 point, very unhealthy: 4 points). Subjective health awareness was reversed to analyze the correlation between other variables. Based on a prior study [9], responses of 'very heathy' and 'healthy' were categorized as positive health awareness, while responses of 'unhealthy' and 'very unhealthy' were categorized as negative health awareness when analyzing a panel logit analysis.

\section{2) Independent variables}

The independent variables of this study were divided into 
environmental, individual, and multicultural characteristics based on the MAPS user guidelines [16].

\section{(1) Environmental characteristics}

The environmental characteristics analyzed as independent variables included residential area and socioeconomic status (measured using monthly income as a proxy).

\section{(2) Personal characteristics}

The personal characteristics analyzed as independent variables included gender, age, body type, drinking and smoking experience, self-esteem, depression, body satisfaction, and social withdrawal. According to the 2017 Korean National Growth Charts for children and adolescents [17], body type was categorized using the body mass index (BMI) based on the 95th percentile cutoffs of the age group. Drinking and smoking experience was classified based on responses of 'I have smoked' and 'I have drunk' in the delinquency experience category. Self-esteem was measured on a 4-point Likert scale (strongly disagree: 1 point, strongly agree: 4 points) with 4 questions, and a higher score indicated higher self-esteem. Depression was measured on a 4-point Likert scale (strongly disagree: 1 point, strongly agree: 4 points) with 10 questions, and a higher score indicated higher depression. Body satisfaction, which is a concept including subjective satisfaction with one's body image and body function [18] was measured on a 4-point Likert scale (strongly disagree: 1 point, strongly agree: 4 points) with 6 questions. Two items ('I want to change some part of my appearance' and 'I am distressed by my appearance') were reversed, and a higher score indicated higher body satisfaction. Social withdrawal refers to difficulties in forming and maintaining social relationships, such as avoidance and isolation [18]. Social withdrawal was measured on a 4-point Likert scale (strongly disagree: 1 point, strongly agree: 4 points) with 5 questions, and a higher score indicated higher level of social withdrawal.

\section{(3) Multicultural characteristics}

Multicultural characteristics included the parental nationality, self-identified nationality (the country to which an individual perceives himself or herself as belonging), experience of visiting the foreign parent's country, interest in the foreign parent's country, acculturative stress, national identity, bicultural acceptance attitude, and multicultural acceptability. Interest in the foreign parent's country was measured on a 5-point Likert scale (no interest: 1 point, very strong interest: 5 points), and a higher score indicated a higher level of interest in the foreign parent's country. Acculturative stress, which refers to difficulties in social, familial, and environmental adaptation due to cultural differences between Korea and the for- eign parent's country $[1,7,16]$, was measured on a 4 -point Likert scale (strongly disagree: 1 point, strongly agree: 4 points) with 10 questions, and a higher score indicated higher acculturative stress. National identity, referring to the degree of emotional involvement with Korea [16,19], was measured on a 4-point Likert scale (strongly disagree: 1 point, strongly agree: 4 points) with 4 questions, and a higher score indicated higher national identity. Bicultural acceptance attitude, which corresponds to a receptive attitude towards Korean culture as the mainstream culture and the foreign parent's country as a non-mainstream culture $[1,16,19]$, was measured on a 4-point Likert scale (strongly disagree: 1 point, strongly agree: 4 points) with 10 questions, and a higher score indicated a higher bicultural acceptance attitude. Multicultural acceptability, the ability to accommodate diverse cultural backgrounds $[16,19]$, was measured on a 4-point Likert scale (strongly disagree: 1 point, strongly agree: 4 points) with 5 questions; a higher score indicated higher multicultural acceptability.

\section{Research Model}

This study performed a panel logit model analysis to identify the factors influencing positive subjective health awareness in multicultural adolescents using Eviews 8.0. When conducting an analysis of cross-sectional data from 3 years (2014-2016), it would be necessary to perform a comparative verification between fixed and random effects through the Hausman test. However, this study did not perform such an analysis because it conducted a panel logit model analysis with panel data from 2016.

\section{1) The effects of environmental and personal characteristics on positive subjective health awareness}

To systematically analyze the effect of environmental and personal characteristics on positive subjective health awareness in multicultural adolescents, a panel logit model was constructed. Positive subjective health awareness (HEA) was used as the dependent variable. As explanatory variables, residential area (ARE), socioeconomic status (SES), gender (GEN), age (AGE), body type (BMI), drinking and smoking experience (DSE), self-esteem (EST), depression (DEP), body satisfaction (BSA), and social withdrawal (WID) were used.

\section{$\mathrm{HEA}=\beta_{0}+\beta_{1} \mathrm{ARE}+\beta_{2} \mathrm{SES}+\beta_{3} \mathrm{GEN}+\beta_{4} \mathrm{AGE}+\beta_{5} \mathrm{BMI}+\beta_{6} \mathrm{DSE}+$ $\beta_{7} \mathrm{EST}+\beta_{8} \mathrm{DEP}+\beta_{9} \mathrm{BSA}+\beta_{10} \mathrm{WID}+\mathrm{e}$}

$\beta_{1}, \beta_{2}, \beta_{3}, \ldots, \beta_{10}$ : coefficient for independent and control variables e: error term

2) The effects of multicultural characteristics on positive subjec- 


\section{tive health awareness}

To systematically analyze the effect of multicultural characteristics on positive subjective health awareness in multicultural adolescents, a panel logit model was constructed. Positive subjective health awareness (HEA) was used as the dependent variable. As explanatory variables, parental nationality (PNT), self-identified nationality (NAT), the experience of visiting the foreign parent's country (VPC), degree of interest in the foreign parent's country (IPC), acculturative stress (ACS), national identity (NAI), bicultural acceptance attitude (BCU), and multicultural acceptability (MUL) were used.

\section{$\mathrm{HEA}=\beta_{0}+\beta_{1} \mathrm{PNT}+\beta_{2} \mathrm{NAT}+\beta_{3} \mathrm{VPC}+\beta_{4} \mathrm{IPC}+\beta_{5} \mathrm{ACS}+\beta_{6} \mathrm{NAI}+$ $\beta_{7} \mathrm{BCU}+\beta_{8} \mathrm{MUL}+\mathrm{e}$}

$\beta_{1}, \beta_{2}, \beta_{3}, \ldots, \beta_{8}$ : coefficient for independent and control variables e: error term

3) The effects of environmental, personal, and multicultural characteristics on positive subjective health awareness

To systematically analyze the effect of environmental, personal, and multicultural characteristics on positive subjective health awareness in multicultural adolescents, a panel logit model was constructed. Positive subjective health awareness (HEA) was used as the dependent variable. The following explanatory variables were used: residential area (ARE), socioeconomic status (SES), gender (GEN), age (AGE), body type (BMI), drinking and smoking experience (DSE), self-esteem (EST), depression (DEP), body satisfaction (BSA), and social withdrawal (WID), parental nationality (PNT), self-identified nationality (NAT), experience of visiting the foreign parent's country (VPC), degree of interest in the foreign parent's country (IPC), acculturative stress (ACS), national identity (NAI), bicultural acceptance attitude (BCU), and multicultural acceptability (MUL).

\section{$\mathrm{HEA}=\beta_{0}+\beta_{1} \mathrm{ARE}+\beta_{2} \mathrm{SES}+\beta_{3} \mathrm{GEN}+\beta_{4} \mathrm{AGE}+\beta_{5} \mathrm{BMI}+\beta_{6} \mathrm{DSE}+$ $\beta_{7} \mathrm{EST}+\beta_{8} \mathrm{DEP}+\beta_{9} \mathrm{BSA}+\beta_{10} \mathrm{WID}+\beta_{10} \mathrm{PNT}+\beta_{10} \mathrm{NAT}+$ $\beta_{10} \mathrm{VPC}+\beta_{10} \mathrm{IPC}+\beta_{10} \mathrm{ACS}+\beta_{10} \mathrm{NAI}+\beta_{10} \mathrm{BCU}+\beta_{10} \mathrm{MUL}+\mathrm{e}$}

$\beta_{1}, \beta_{2}, \beta_{3}, \ldots, \beta_{18}$ : coefficient for independent and control variables e: error term

\section{RESULTS}

\section{Descriptive Statistics}

The descriptive statistics for the multicultural adolescents are shown in Table 1 . The overwhelming majority $(95.3 \%)$ of subjects in this study had positive subjective health aware- ness. In terms of environmental characteristics, $71.1 \%$ of the subjects lived in metropolitan or medium/small cities, and the average monthly income was 2,100 dollars. Approximately half $(49.2 \%)$ of the subjects were male, and $88.6 \%$ of subjects were 15 years old. Furthermore, $92.5 \%$ of the subjects were non-obese and $95.9 \%$ of them did not have drinking or smoking experience. The mean score for self-esteem was $3.13 \pm 0.54$, and that for depression was $1.71 \pm 0.53$. The mean score for body satisfaction was $3.02 \pm 0.50$, and that for social withdrawal was $2.32 \pm 0.72$. Regarding multicultural characteristics, $96.5 \%$ of subjects were children of foreign mothers, and $73.9 \%$ of them recognized themselves as Koreans. Additionally, 81.1\% of subjects had an experience of visiting the foreign parent's country, and the subjects had a moderate level of interest in the foreign parent's country $(3.50 \pm 0.91)$. The mean score for acculturative stress was $1.41 \pm 0.32$, and that for national identity was $2.82 \pm 0.64$. The mean score for bicultural acceptance attitude was $2.92 \pm 0.38$, and that for multicultural acceptability was $3.14 \pm 0.54$.

\section{Correlations between Subjective Health Awareness and Other Variables}

The correlations between subjective health awareness and self-esteem, depression, body satisfaction, social withdrawal, interest in the foreign parent's country, acculturative stress, national identity, bicultural acceptance attitude, and multicultural acceptability are shown in Table 2 . Among personal characteristics, self-esteem $(\mathrm{r}=.29 . p<.001)$ and body satisfaction $(\mathrm{r}=.20, p<.001)$ showed significant positive correlations with subjective health awareness, while depression $(\mathrm{r}=-.31$, $p<.001)$ and social withdrawal $(\mathrm{r}=-.20, p<.001)$ showed significant negative correlations. Among the multicultural characteristics, national identity $(\mathrm{r}=.16, p<.001)$, bicultural acceptance attitude $(\mathrm{r}=.16, p<.001)$, and multicultural acceptability $(\mathrm{r}=.11, p<.001)$ showed significant positive correlations with subjective health awareness, while acculturative stress showed a significant negative correlation $(\mathrm{r}=-.16, p<.001)$.

\section{The Effects of Environmental and Personal Characteris- tics on Positive Subjective Health Awareness}

The effects of environmental and personal characteristics on positive subjective health awareness are shown in Table 3. Socioeconomic level $(\mathrm{r}=.02, \mathrm{t}=2.06, p=.039)$ had a significant positive effect on positive subjective health awareness, while depression $(\mathrm{r}=-.82, \mathrm{t}=2.66, p=.007)$ had a significant negative effect.

The panel logit model using these characteristics showed an explanatory power of $9 \%$ (McFadden=.09), and the log- 
Table 1. Descriptive Statistics $(N=1,329)$

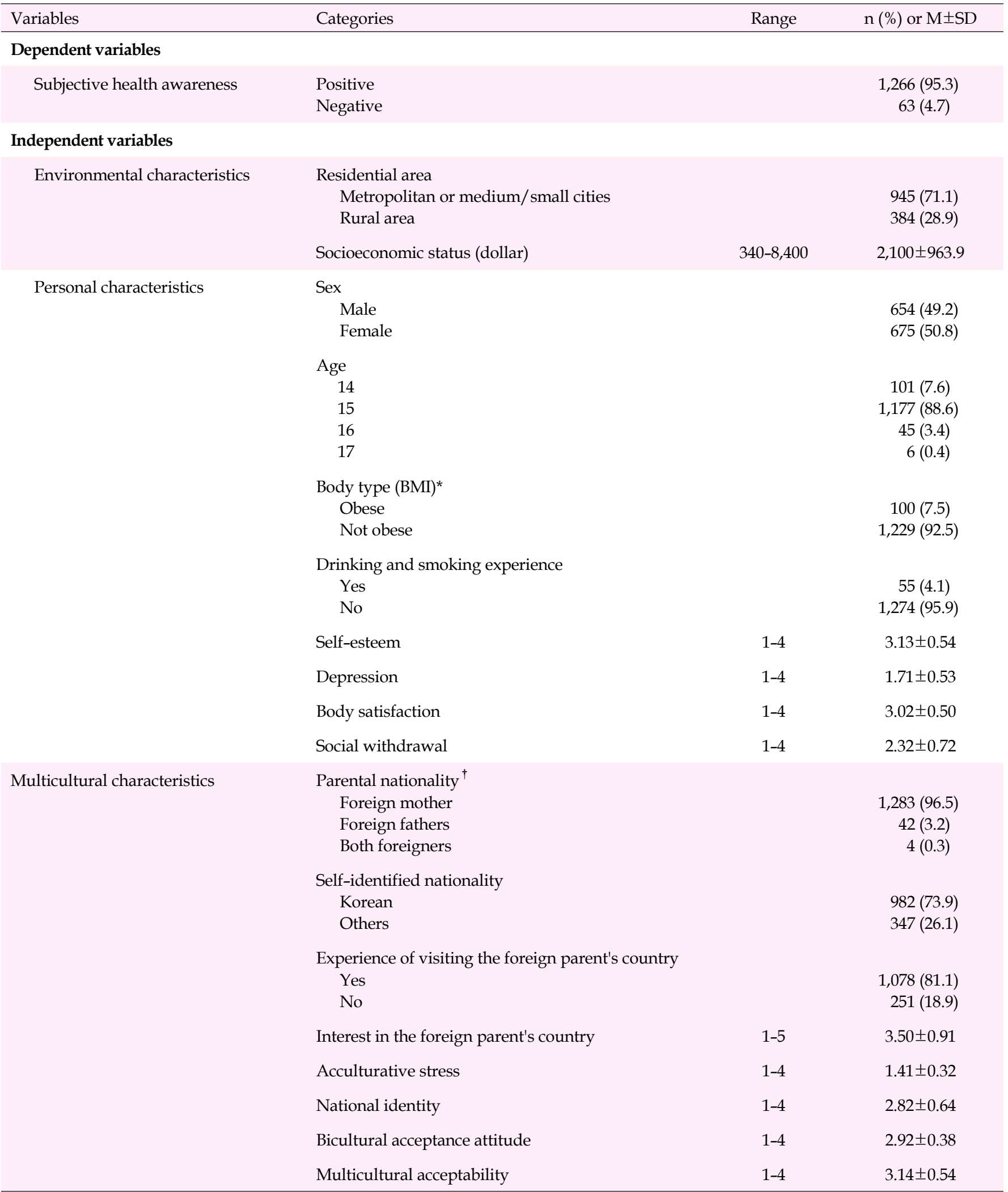

*BMI $\left(\mathrm{kg} / \mathrm{m}^{2}\right)$ based on the 95th percentile cutoff: 14-year-old boys $\geq 26.0$, 14-year-old girls $\geq 25.2$, 15-year-old boys $\geq 26.2$, 15-year-old girls $\geq 25.4$, 16 -year-old boys $\geq 26.4,16$-year-old girls $\geq 25.5,17$-year-old boys $\geq 26.6,17$-year-old girls $\geq 25.5 ;{ }^{\dagger}$ In the raw data, the foreign status of parents was categorized into foreign mother, foreign father, both foreigners, but it was converted to a dummy scale during logit regression; BMI, body mass index. 
Table 2. Correlations between Subjective Health Awareness and Other Variables $(N=1,329)$

\begin{tabular}{|c|c|c|c|c|c|c|c|c|c|}
\hline \multirow{3}{*}{ Variables } & \multicolumn{4}{|c|}{ Personal characteristics } & \multicolumn{5}{|c|}{ Multicultural characteristics } \\
\hline & $\begin{array}{c}\text { Self- } \\
\text { esteem }\end{array}$ & Depression & $\begin{array}{c}\text { Body } \\
\text { satisfaction }\end{array}$ & $\begin{array}{c}\text { Social } \\
\text { withdrawal }\end{array}$ & $\begin{array}{l}\text { Interest in } \\
\text { the foreign } \\
\text { parent's } \\
\text { country }\end{array}$ & $\begin{array}{c}\text { Acculturative } \\
\text { stress }\end{array}$ & $\begin{array}{l}\text { National } \\
\text { identity }\end{array}$ & $\begin{array}{l}\text { Bicultural } \\
\text { acceptance } \\
\text { attitude }\end{array}$ & $\begin{array}{l}\text { Multicultural } \\
\text { acceptability }\end{array}$ \\
\hline & $\mathrm{r}(p)$ & $\mathrm{r}(p)$ & $\mathrm{r}(p)$ & $\mathrm{r}(p)$ & $\mathrm{r}(p)$ & $\mathrm{r}(p)$ & $\mathrm{r}(p)$ & $\mathrm{r}(p)$ & $\mathrm{r}(p)$ \\
\hline $\begin{array}{l}\text { Subjective health } \\
\text { awareness }\end{array}$ & $\begin{array}{c}.29 \\
(<.001)\end{array}$ & $\begin{array}{c}-.31 \\
(<.001)\end{array}$ & $\begin{array}{c}.20 \\
(<.001)\end{array}$ & $\begin{array}{c}-.20 \\
(<.001)\end{array}$ & $\begin{array}{c}.01 \\
(.630)\end{array}$ & $\begin{array}{c}-.16 \\
(<.001)\end{array}$ & $\begin{array}{c}.16 \\
(<.001)\end{array}$ & $\begin{array}{c}.16 \\
(<.001)\end{array}$ & $\begin{array}{c}.11 \\
(<.001)\end{array}$ \\
\hline
\end{tabular}

Table 3. The Effects of Environmental and Personal Characteristics on Positive Subjective Health Awareness $(N=1,329)$

\begin{tabular}{|c|c|c|c|c|c|}
\hline Variables & Categories & Coefficient & SE & Z-Statistic (t) & $p$ \\
\hline \multicolumn{6}{|l|}{ Environmental characteristics } \\
\hline Residential area & $\begin{array}{l}\text { Metropolitan or medium/small cities } \\
\text { Rural area (ref.) }\end{array}$ & -.18 & 0.31 & 0.58 & .561 \\
\hline Socioeconomic status & & .02 & 0.00 & 2.06 & .039 \\
\hline \multicolumn{6}{|l|}{ Personal characteristics } \\
\hline Gender & $\begin{array}{l}\text { Male } \\
\text { Female (ref.) }\end{array}$ & -.08 & 0.27 & 0.28 & .778 \\
\hline Age & & -.16 & 0.35 & 0.45 & .655 \\
\hline Body type (BMI)* & $\begin{array}{l}\text { Obese } \\
\text { Not obese (ref.) }\end{array}$ & -.02 & 0.62 & 0.03 & .980 \\
\hline $\begin{array}{l}\text { Drinking and smoking } \\
\text { experience }\end{array}$ & $\begin{array}{l}\text { Yes } \\
\text { No (ref.) }\end{array}$ & -.23 & 0.56 & 0.42 & .677 \\
\hline Self-esteem & & .26 & 0.32 & 0.79 & .429 \\
\hline Depression & & -.82 & 0.31 & 2.66 & .007 \\
\hline Body satisfaction & & .60 & 0.36 & 1.68 & .093 \\
\hline Social withdrawal & & -.15 & 0.23 & 0.63 & .525 \\
\hline
\end{tabular}

${ }^{*}$ BMI $\left(\mathrm{kg} / \mathrm{m}^{2}\right)$ based on the 95th percentile cutoff: 14-year-old boys $\geq 26.0,14$-year-old girls $\geq 25.2,15$-year-old boys $\geq 26.2,15$-year-old girls $\geq 25.4$, 16-year-old boys $\geq 26.4$, 16-year-old girls $\geq 25.5$, 17-year-old boys $\geq 26.6$, 17-year-old girls $\geq 25.5$; BMI, body mass index; df, degrees of freedom; LR, likelihood ratio; ref., reference; SE, standard error.

likelihood was -228.29. This panel logit model was statistically significant (likelihood-ratio [LR] statistic $=42.72, p<.001$ ).

\section{The Effects of Multicultural Characteristics on Positive Subjective Health Awareness}

The effects of multicultural characteristics on positive subjective health awareness are shown in Table 4 . National identity $(\mathrm{r}=.46, \mathrm{t}=2.15, p=.031)$ had a significant positive effect on positive subjective health awareness.

The panel logit model constructed using these characteristics had an explanatory power of 3\% (McFadden=.03), and the log-likelihood was -246.30. This panel logit model was statistically significant (LR statistic $=14.54, p<.001$ )

\section{Factors Influencing Positive Subjective Health Aware- ness}

The effects of environmental, personal, and multicultural characteristics on positive subjective health awareness are shown in Table 5. Socioeconomic status $(\mathrm{r}=.02, \mathrm{t}=2.13, p=.033)$ and body satisfaction $(\mathrm{r}=.71, \mathrm{t}=1.97, p=.048)$ had significant positive effects on positive subjective health awareness, whereas depression $(\mathrm{r}=-.74, \mathrm{t}=2.35, p=.018)$ had a significant negative effect.

The panel logit model using environmental, personal, and multicultural characteristics had an explanatory power of $10 \%$ (McFadden $\mathrm{R}^{2}=.10$ ), and the log-likelihood was -225.40 . This panel logit model was statistically significant $($ LR statistic $=48.51$, $p<.001)$. 
Table 4. The Effects of Multicultural Characteristics on Positive Subjective Health Awareness $(N=1,329)$

\begin{tabular}{|c|c|c|c|c|c|}
\hline Variables & Categories & Coefficient & SE & Z-Statistic $(\mathrm{t})$ & $p$ \\
\hline Parental nationality & $\begin{array}{l}\text { Foreign mother } \\
\text { Others (ref.) }\end{array}$ & -.25 & 0.75 & 0.33 & .738 \\
\hline Self-identified nationality & $\begin{array}{l}\text { Korean } \\
\text { Others (ref.) }\end{array}$ & .33 & 0.31 & 1.04 & .296 \\
\hline Experience of visiting the foreign parent's country & $\begin{array}{l}\text { Yes } \\
\text { No (ref.) }\end{array}$ & .16 & 0.32 & 0.48 & .629 \\
\hline Interest in the foreign parent's country & & -.18 & 0.17 & 1.07 & .282 \\
\hline Acculturative stress & & -.48 & 0.37 & 1.31 & .190 \\
\hline National identity & & .46 & 0.21 & 2.15 & .031 \\
\hline Bicultural acceptance attitude & & .55 & 0.41 & 1.34 & 179 \\
\hline Multicultural acceptability & & -.04 & 0.26 & 0.15 & .880 \\
\hline
\end{tabular}

McFadden $\mathrm{R}^{2}=.03$, Log likelihood=-246.30, Akaike information criterion=0.38, LR Statistic $(8 \mathrm{df})=14.54$, Schwarz criterion $=0.42, p$ for $\mathrm{LR}<.001$

df, degrees of freedom; LR, likelihood ratio; ref., reference; SE, standard error.

Table 5. The Effects of Environmental, Personal, and Multicultural Characteristics on Positive Subjective Health Awareness $(N=1,329)$

\begin{tabular}{|c|c|c|c|c|c|}
\hline Variables & Categories & Coefficient & SE & Z-Statistic (t) & $p$ \\
\hline Sex & $\begin{array}{l}\text { Male } \\
\text { Female (ref.) }\end{array}$ & -.10 & 0.28 & 0.35 & .729 \\
\hline Age & & -.13 & 0.35 & 0.36 & .718 \\
\hline Residential area & $\begin{array}{l}\text { Metropolitan or medium/small cities } \\
\text { Rural area (ref.) }\end{array}$ & -.15 & 0.31 & 0.48 & .632 \\
\hline Body type (BMI)* & $\begin{array}{l}\text { Obese } \\
\text { Not obese (ref.) }\end{array}$ & .00 & 0.63 & 0.06 & .950 \\
\hline Socioeconomic status & & .02 & 0.00 & 2.13 & .033 \\
\hline Drinking and smoking experience & $\begin{array}{l}\text { Yes } \\
\text { No (ref.) }\end{array}$ & -.31 & 0.56 & 0.55 & .585 \\
\hline Self-esteem & & .22 & 0.33 & 0.65 & .516 \\
\hline Body satisfaction & & .71 & 0.36 & 1.97 & .048 \\
\hline Depression & & -.74 & 0.32 & 2.35 & .018 \\
\hline Social withdrawal & & -.10 & 0.23 & 0.44 & .663 \\
\hline Parental nationality & $\begin{array}{l}\text { Foreign mother } \\
\text { Others (ref.) }\end{array}$ & -.27 & 0.77 & 0.36 & .722 \\
\hline Self-identified nationality & $\begin{array}{l}\text { Korean } \\
\text { Others (ref.) }\end{array}$ & .16 & 0.33 & 0.49 & .624 \\
\hline $\begin{array}{l}\text { Experience of visiting the foreign } \\
\text { parent's country }\end{array}$ & $\begin{array}{l}\text { Yes } \\
\text { No (ref.) }\end{array}$ & .05 & 0.34 & 0.15 & .881 \\
\hline Interest in the foreign parent's country & & -.17 & 0.17 & 0.96 & .335 \\
\hline Acculturative stress & & .11 & 0.43 & 0.26 & .794 \\
\hline National identity & & .37 & 0.22 & 1.69 & .091 \\
\hline Bicultural acceptance attitude & & .08 & 0.44 & 0.18 & .860 \\
\hline Multicultural acceptability & & -.19 & 0.28 & 0.69 & .491 \\
\hline
\end{tabular}

McFadden $\mathrm{R}^{2}=0.10$, Log likelihood=-225.40, Akaike information criterion=0.37, LR Statistic $(18 \mathrm{df})=48.51$, Schwarz criterion=0.45, $p$ for LR $<.001$.

${ }^{*}$ BMI $\left(\mathrm{kg} / \mathrm{m}^{2}\right)$ based on the 95th percentile cutoff: 14-year-old boys $\geq 26.0,14$-year-old girls $\geq 25.2,15$-year-old boys $\geq 26.2,15$-year-old girls $\geq 25.4$, 16-year-old boys $\geq 26.4$, 16-year-old girls $\geq 25.5$, 17-year-old boys $\geq 26.6$, 17-year-old girls $\geq 25.5$; BMI, body mass index; df, degrees of freedom; LR, likelihood ratio; ref., reference; SE, standard error. 


\section{DISCUSSION}

This study used raw data from the fourth (2014) to the sixth (2016) waves of the MAPS [16] to identify the influence of environmental, personal, and multicultural characteristics on positive subjective health awareness in multicultural adolescents of middle school age.

Most subjects recognized themselves as healthy, which is similar to the findings of a prior study [12] on non-multicultural adolescents. Adolescents are generally in excellent health and have few diseases; thus, they tend to perceive their health positively $[9,20]$. The average household monthly income was 2,100 dollars, which is lower than the median household income of 3,698 dollars (4.39 million won) in 2016 [21]. A similar result was found in a prior study $[2,6]$ reporting that the economic level of multicultural adolescents was significantly lower than that of non-multicultural adolescents. The mean self-esteem score $(3.13 \pm 0.54)$ was lower than those reported by Choi et al. [22] and Lee et al. [23] in studies of non-multicultural adolescents using panel data. The mean score for depression $(1.71 \pm 0.53)$ was higher than those reported by Choi et al. [22] and Lee et al. [23]. This result can be interpreted as indicating that multicultural adolescents experience higher levels of depression, which may be related to their unique multicultural characteristics as well as aspects of physical and psychological development in adolescence [1,7]. The mean score for social withdrawal $(2.32 \pm 0.72)$ was higher than that found in a study conducted among non-multicultural adolescents [22]. This result may be due to social prejudice and distorted perceptions of multicultural families, which may cause multicultural experience more social withdrawal than non-multicultural adolescents [18]. As multicultural adolescents become older, their self-esteem decreases, while depression and social withdrawal increase [24]; thus, nursing interventions and educational programs that enhance the psychological status of multicultural adolescents are recommended. Regarding multicultural characteristics, most subjects were children of foreign mothers. Children of international marriage families account for the highest percentage of multicultural adolescents in Korea, and that was the case for most subjects in this study [16]. The mean score for acculturative stress $(1.41 \pm 0.32)$ was higher than has been reported in prior study [1] conducted among multicultural adolescents in the first year of middle school. However, this score was lower than that found by Kim et al. [18] in a study including high school students. This finding can be interpreted as indicating that the acculturative stress of multicultural adolescents increases over time [24]. The mean scores for national identity $(2.82 \pm 0.64)$ and bicultural acceptance attitude $(2.92 \pm 0.38)$ were similar to those found by Lee and Choi [19]. National identity and bicultural acceptance attitude decrease as multicultural adolescents become older [24]; thus, further research on multicultural characteristics over time is needed.

The correlation analysis showed that subjective health awareness was related to self-esteem, depression, body satisfaction, and social withdrawal among personal characteristics, and acculturative stress, national identity, bicultural acceptance attitude, and multicultural acceptability among multicultural characteristics. The relationships between subjective health awareness and personal characteristics can be interpreted as indicating that physical, social, and mental function and state are central to adolescents' perceptions of their health $[9,20]$. In addition, the relationship between multicultural characteristics and subjective health awareness can be explained as showing that sociocultural factors and ideologies affect the concept of health and health awareness, and the mechanisms of recognizing and solving health problems differ depending on ethnicity and race [14]. Among the environmental characteristics, socioeconomic status showed an association with positive subjective health awareness. Socioeconomic status is a factor influencing one's subjective health condition, and adolescents are sensitive to socioeconomic indicators in judging their health status $[1,8,9]$. The health status of adolescents is also related to the structural characteristics of the family, which affect the child-rearing conditions, and environmental factors that determine health behavior and management [4,12]. Turning to personal characteristics, depression showed an inverse relationship with positive subjective health awareness. This finding is similar to the results of a previous study [11,12] conducted on non-multicultural adolescents, which found that adolescents with depression perceived themselves as unhealthy. These findings can be interpreted as showing that depression influences health $[8,11,12]$ and that psychological factors are the main factors that affect subjective health awareness in adolescence $[9,11,12,20]$. In this study, self-esteem was not related to positive subjective health awareness. This stands in contrast with a result from a prior study [25] conducted on non-multicultural adolescents abroad. Self-esteem induces positive health behavior through positive health awareness [26]. Since the mean score for self-esteem in this study was lower than that found in the above-mentioned prior study [25], nursing education and interventions to improve self-esteem in multicultural adolescents are recommended. National identity is a psychological state, but it affects all aspects of individual life, such as an individual's subjective well-being and physical/ mental health; thus, individuals with high national identity positively evaluate themselves and the group to which they belong $[19,27]$. Thus, it is thought that multicultural adolescents with high national identity are more likely to perceive 
themselves as healthy.

The panel logit model analysis showed that socioeconomic status, body satisfaction, and depression had significant effects on positive subjective health awareness. Adolescence is the intermediate period in the transition from childhood to adulthood, wherein adolescents pay closer attention to their own and their families' economic condition [8,9], and the subjective health awareness of adolescents is mostly determined by personal factors [12]. Adolescents experience rapid growth and are sensitive to their appearance and body; thus, body satisfaction is related to psychological development and mental health [18]. Adolescents with high body satisfaction tend to have positive body image and self-concept, so they perceive themselves as healthy [28]. Thus, it can be interpreted that the effect of environmental and personal characteristics was relatively high in this study. The multicultural characteristics that were significant in model 2 were not significant in model 3. This result is similar to that of a prior study [29], which found that multicultural characteristics had smaller effects than demographic and personal characteristics on adolescents' mental status. Multicultural characteristics, such as bicultural stress and discrimination experience, influenced and predicted personal characteristics such as depression, social withdrawal, and self-esteem [30]. In addition, multicultural characteristics cause differences in environmental characteristics such as environmental setting, medical care, and socioeconomic status [30]. These considerations may explain why the influence of multicultural characteristics was offset in model 3. Further studies on the relationship of multicultural characteristics to the environmental and personal characteristics of multicultural adolescents are recommended.

This study is meaningful in that various aspects of environmental, personal, and multicultural characteristics were investigated using representative data from the MAPS [16]. However, this study has the following limitations. First, the study was conducted only among multicultural adolescents and did not involve a comparison with non-multicultural adolescents. Thus, further studies comparing both groups are recommended. Second, this study did not include a fully comprehensive range of variables due to the utilization of secondary data, and the explanatory power of the findings was low. Therefore, additional research on factors influencing positive subjective health awareness should include other variables such as exercise, sleeping habits, and eating habits. Third, subjective health awareness in this study was measured with one question. Since subjective health awareness was found to be influenced by environmental, personal, and multicultural characteristics, the development of a multidimensional tool is suggested. Finally, this study was conducted on multicultural adolescents of middle school age. Although both middle- and high-school students are in adolescence, their maturity and sensitivity differ [5]. Thus, further studies of subjective health awareness among high-school-age subjects are recommended.

\section{CONCLUSION}

This study used raw data from the MAPS [16] to identify factors influencing positive subjective health awareness in multicultural adolescents. Positive subjective health awareness was positively related to socioeconomic status as an environmental characteristic, negatively related to depression as a personal characteristic, and positively related to national identity as a multicultural characteristic. Socioeconomic status, body satisfaction, and depression showed significant effects on positive subjective health awareness. When multicultural characteristics were entered into a research model with environmental and personal characteristics, it was found that multicultural characteristics did not significantly affect positive subjective health awareness.

The following suggestions are made based on the results of this study. From an environmental perspective, economic support to improve the structural environment of multicultural adolescents is recommended. From a personal perspective, nursing interventions and educational programs that improve the psychological status of multicultural adolescents are recommended. From a multicultural perspective, it is necessary for nurses to be sensitive to multicultural adolescents' cultural backgrounds. Finally, since environmental and personal characteristics were found to have a stronger influence than multicultural characteristics, nurses need to focus on the developmental characteristics of adolescence rather than taking a discriminatory approach when providing care to multicultural adolescents to foster positive awareness of their health.

\section{ORCID}

Sun Yeob Choi ～https://orcid.org/0000-0001-9879-2947

\section{Authors' contribution}

Conceptualization: Sun Yeob Choi; Data curation, Formal analysis: Sun Yeob Choi; Writing-original draft, Writing-review and editing: Sun Yeob Choi; Final approval of published version: Sun Yeob Choi.

\section{Conflict of interest}

No existing or potential conflict of interest relevant to this article was reported. 


\section{Funding}

None.

\section{Data availability}

Please contact the corresponding author for data availability.

\section{Acknowledgements}

This study used raw data from the fourth wave (2014) to the sixth wave (2016) of the Multicultural Adolescents Panel Study conducted by the National Youth Policy Institute.

\section{REFERENCES}

1. Eun S, Lee S, Lee K. Acculturative stress amongst multicultural adolescents: patterns, predictors, and psychosocial outcomes. Studies on Korean Youth. 2019;30(2):177-211.

https://doi.org/10.14816/sky.2019.30.2.177

2. Kim MK. A convergent study on factors influencing adolescents' mental health of multicultural family. Journal of the Korea Convergence Society. 2018;9(1):187-197.

https://doi.org/10.15207/JKCS.2018.9.1.189

3. Statistics Korea. 2019 Adolescent statistics [Internet]. Daejeon: Statics Korea; 2019 [cited 2021 August 2]. Available from: http://kostat.go.kr/portal/korea/kor_nw/1/6/1/index.board? bmode $=$ read\&aSeq $=374490 \&$ page $\mathrm{No}=\&$ row $\mathrm{Num}=10 \& a m S e q=\&$ sTarget $=\&$ s $x t$

4. Park SJ. The longitudinal effects of youth social capital on self-rated health. Studies on Korean Youth. 2018;29(2):241-269.

https://doi.org/10.14816/sky.2018.29.2.241

5. Kim SJ, Han JA, Kim EJ, Lee SY. Association between sleep insufficient type and suicidal ideation among Korean middle and high school student. Korean Journal of Health Education and Promotion. 2021;38(2):15-27. https://doi.org/10.14367/kjhep.2021.38.2.15

6. Chae MO. Subjective health status, mental health and internet addiction tendency of adolescents in multi-cultural families compared to general families. Journal of Digital Convergence. 2018;16(12): 383-393. https://doi.org/10.14400/JDC.2018.16.12.383

7. Ann HW, Um MY. The mediating effect of depression in the impact of cultural adaptation stress on school life adaptation of multicultural adolescents: Searching for converged intervention strategies. Journal of Korea Convergence Society. 2020;11(6):297-310. https://doi.org/10.15207/JKCS.2020.11.6.297

8. Shin J, Lee H, Choi EK, Nam C, Chae SM, Park O. Social determinants of health and well-being of adolescents in multicultural families in South Korea: Social-cultural and community influence. Frontiers in Public Health. 2021;9:641140. https://doi.org/10.3389/fpubh.2021.641140

9. Choi KW. Factors related to self-rated health in adolescents: Findings from the Korea Youth Panel Survey. Korean Journal of Health Education and Promotion. 2014;31(3):39-50.

https://doi.org/10.14367/kjhep.2014.31.3.39

10. Nam SI, Choi K, Yi HJ. Self-perceived health status, depression, and suicidal behavior among people with diabetes. Korean Journal of Social Welfare Studies. 2014;45(1):231-254.

https://doi.org/10.16999/kasws.2014.45.1.231

11. An J, Kim H. A study on the determinants of children and adolescents' health inequality in Korea. Studies on Korean Youth. 2013;24(2):205-231.

12. Lee JH, Yi KH. An analysis of the determinants of adolescents' health inequality in Korea: A view from the students' self-rated health condition and obesity. Korea Educational Review. 2015;21(2):217-243.

13. Oh SH. The study on the eco-systemic variables affecting multicultural family adolescents' depression. Journal of Community Welfare. 2016;56(1):1-27. https://doi.org/10.15300/jcw.2016.56.1.1

14. Stepanikova I, Oates GR. Dimensions of racial identity and perceived discrimination in health care. Ethnicity and Disease. 2016; 26(4):501-512. https://doi.org/10.18865/ed.26.4.501

15. Yoo BN, Park KO, Choi JY. Association between self-esteem and health behavior of the children with multi-cultural family background. Journal of Korean Society for School and Community Health Education. 2010;11(1):41-55.

16. National Youth Policy Institute. Multicultural Adolescents Panel Study: 1st-8th wave data user guide [Internet]. Sejong: National Youth Policy Institute Korea; 2020 [cited 2021 March 3]. Available from: http://www.nypi.re.kr

17. Kim JH, Yun S, Hwang S, Shim JO, Chae HW, Lee YJ, et al. The 2017 Korean national growth charts for children and adolescents: Development, improvement, and prospects. Korean Journal of Pediatrics. 2018;61(5):135-149. https://doi.org/10.3345/kjp.2018.61.5.135

18. Kim DS, Park BS, Lee EB, Sohn GH. The influence of body-cathexis on depression among adolescent of the multi-cultural family: Focus on the mediating effect of social withdrawal. Korean Journal of Social Welfare Research. 2020;64:91-125.

https://doi.org/10.17997/SWRY.64.1.4

19. Lee $\mathrm{SE}$, Choi N. The mediating role of bicultural experience in the relationship between self-esteem and life satisfaction of multicultural adolescents. Journal of Families and Better Life. 2021;39(1): 17-29. https://doi.org/10.7466/JFBL.2021.39.1.17

20. Mechanic D, Hansell S. Adolescent competence, psychological well-being, and self-assessed physical health. Journal of Health and Social Behavior. 1987;28(4):364-374. https://doi.org/10.2307/2136790

21. Statistics Korea. Report on the survey of household finances and living conditions [Internet]. Daejeon: Statics Korea; 2016 [cited 
2021 September 4]. Available from:

https://www.index.go.kr/potal/main/EachDtlPageDetail.do?idx cd $=2762$

22. Choi W, Kang S, Paeg S. The effects of middle school students' depression and anxiety on suicide accidents: Mediated moderation effect of atrophy and self-esteem. Forum for Youth Culture. 2020; 63(0):85-108. https://doi.org/10.17854/ffyc.2020.07.63.85

23. Lee BJ, Son SO, Kim YJ. The effects of school-related stress on depression among adolescents and the moderating effects of selfesteem. Journal of School Social Work. 2015;31:37-58.

24. Kim HC, Mo SH, Oh SB, Kim SH, Baek SE. Longitudinal survey of multicultural youth and study on policy measures III. Survey Report. Sejong: National Youth Policy Institute; 2015. Report No.: 15-R13.

25. Waters EB, Salmon LA, Wake M, Wright M, Hesketh KD. The health and well-being of adolescents: A school-based population study of the self-report Child Health Questionnaire. Journal of Adolescent Health. 2001;29(2):140-149. https://doi.org/10.1016/s1054-139x(01)00211-7

26. Yarcheski A, Mahon NE, Yarcheski TJ. Alternative models of positive health practices in adolescents. Nursing Research. 1997;46(2): 85-92. https://doi.org/10.1097/00006199-199703000-00005

27. Kim HJ, Yoo HY. Determinants of national identity among North Korean refugees. Journal of Eurasian Studies. 2014;11(3):45-71. https://doi.org/10.31203/aepa.2014.11.3.003

28. Kim DJ. Relationship among physical self-concept, perceived health level and happiness in teenagers. The Journal of Namseoul University. 2010;16(2):173-184.

29. Yang SY, Park SK, Kim MS. Effects of bicultural characteristics and social capital on psychological adaptation. Journal of the Korea Contents Association. 2013;13(6):270-282. https://doi.org/10.5392/JKCA.2013.13.06.270

30. Pachter LM, Coll CG. Racism and child health: A review of the literature and future directions. Journal of Developmental and Behavioral Pediatrics. 2009;30(3):255-263. https://doi.org/10.1097/DBP.0b013e3181a7ed5a 